\title{
Estrategias de estimulación temprana dentro de unidades de neonatología para bebés pretérmino ${ }^{1}$
}

Institución: Universidad de Costa Rica, Maestría en Enfermería Pediátrica

\author{
José Ortega Matarrita ${ }^{2}$
}

Carolina Núñez Chaves ${ }^{3}$

\section{RESUMEN}

Debido a la mejora constante de los servicios de neonatología, se logra una mayor sobrevida de los bebés prematuros; no obstante, su grado de inmadurez los coloca en una situación de fragilidad que los expone tanto a una mayor morbilidad, como a retrasos en el desarrollo, con repercusiones a corto, mediano y largo plazo. Los servicios de neonatología, en ocasiones de Unidad de Cuidados Intensivos Neonatales, son el primer contacto del recién nacido al momento del nacimiento, donde es sometido a múltiples estímulos ambientales que interfieren en su desarrollo. El objetivo de este estudio es analizar la mejor evidencia científica disponible respecto de las estrategias de estimulación temprana aplicadas por enfermería a los recién nacidos pretérmino, hospitalizados, que han sido útiles para reducir o evitar alteraciones leves o graves en el neurodesarrollo. Respecto de la metodología para desarrollar este protocolo se aplicará la práctica clínica basada en evidencia, que consta de cinco pasos: la pregunta clínica, búsqueda de evidencia científica, análisis crítico de la evidencia, transferencia de la evidencia y evaluación de la transferencia de la evidencia.

Palabras claves: enfermería-pediátrica, estimulación-temprana, neurodesarrollo, recién-nacido-pretérmino

\footnotetext{
${ }^{1}$ Fecha de recibido: 5 de diciembre del 2017

Fecha de aceptación: 16 de marzo del 2018

${ }^{2}$ Enfermero, estudiante de maestría en Enfermería Pediátrica con énfasis en Neonatología. Hospital Adolfo Carit Eva. Universidad de Costa Rica, Costa Rica. Correo electrónico: jose88carit@gmail.com

${ }^{3}$ Enfermera, estudiante de maestría en Enfermería Pediátrica con énfasis en Neonatología. Hospital Nacional de Niños. Universidad de Costa Rica, Costa Rica. Correo electrónico: carola ch11@,hotmail.com
} 


\title{
Early stimulation strategies within Neonatal Units for preterm babies ${ }^{1}$
}

Institution: University of Costa Rica, Postgraduate Pediatric Nursing

José Ortega Matarrita ${ }^{2}$

Carolina Núñez Chaves ${ }^{3}$

\begin{abstract}
Due to the constant improvement of neonatology services, a greater survival of premature babies is achieved, however, their degree of immaturity places them in a situation of fragility that exposes them to greater morbidity, as well as delays in development, with repercussions in the short, medium and long term. The neonatal services, sometimes in the Neonatal Intensive Care Unit, are the first contact of the newborn at birth, being subjected to multiple environmental stimuli that interfere in its development. The aim of this study is to analyze the best available scientific evidence regarding the strategies of early stimulation applied by nursing to hospitalized preterm newborns, which have been useful to reduce or avoid mild or severe alterations in neurodevelopment. Regarding the methodology to develop this protocol, clinical practice based on evidence will be applied, consisting of five steps; the clinical question, search for scientific evidence, critical analysis of the evidence, transfer of evidence and evaluation of the transfer of evidence.
\end{abstract}

Keywords: nursing-pediatric, early-stimulation, neurodevelopment, new-born-preterm

\footnotetext{
${ }^{1}$ Date of receipt: December 5, 2017

Date of acceptance: March 16, 2018

${ }^{2}$ Nurse, Master's student in Pediatric Nursing with an emphasis in Neonatology. Hospital Adolfo Carit Eva. University of Costa Rica, Costa Rica. E-mail: jose88carit@gmail.com

${ }^{3}$ Nurse, Master's student in Pediatric Nursing with an emphasis in Neonatology. National Children's Hospital. University of Costa Rica, Costa Rica. E-mail: carola ch11@,hotmail.com
} 


\section{Estratégias de estimulação precoce dentro de unidades neonatais para bebês prematuros ${ }^{1}$}

José Ortega Matarrita ${ }^{2}$

Carolina Núñez Chaves ${ }^{3}$

Instituição: Universidade da Costa Rica, Enfermagem pediátrica de pós-graduação

\section{RESUMO}

Devido à melhoria constante dos serviços de neonatologia, uma maior sobrevivência dos bebês prematuros é alcançada, no entanto, seu grau de imaturidade coloca-os em uma situação de fragilidade que os expõe a uma maior morbidade, além de atrasos no desenvolvimento, com repercussões no curto, médio e longo prazo. Os serviços neonatais, às vezes na Unidade de Cuidados Intensivos Neonatais, são o primeiro contato do recém nascido no nascimento, sendo submetidos a múltiplos estímulos ambientais que interferem no seu desenvolvimento. O objetivo deste estudo é analisar a melhor evidência científica disponível sobre as estratégias de estimulação precoce aplicadas pela enfermagem a recém-nascidos prematuros hospitalizados, que foram úteis para reduzir ou evitar alterações leves ou graves no desenvolvimento neurológico. Em relação à metodologia para desenvolver este protocolo, será aplicada prática clínica baseada em evidências, consistindo em cinco etapas; a questão clínica, a busca de evidências científicas, a análise crítica da evidência, a transferência de evidências e a avaliação da transferência de provas.

Palavras-chave: enfermagem-pediatria, estimulação-precoce, neurodesenvolvimento, recém-nascido-pré-termo

\footnotetext{
${ }^{1}$ Data de recepção: 5 de dezembro de 2017

Data de aceitação: 16 de março de 2018 ${ }^{2}$ Enfermeiro, aluna de mestrado em enfermagem pediátrica com ênfase em neonatologia. Hospital Adolfo Carit Eva. Universidade da Costa Rica, Costa Rica. Correio eletrônico: jose88carit@gmail.com

${ }^{3}$ Enfermeira, aluna de mestrado em enfermagem pediátrica com ênfase em neonatologia. Hospital Nacional da Criança. Universidade da Costa Rica, Costa Rica. Correio eletrônico: carola ch11@hotmail.com
} 


\section{Revista Electrónica Enfermeria Actual en costa Rica}

\section{INTRODUCCIÓN}

Durante los últimos veinte años, los grandes adelantos en la atención de enfermería de los recién nacidos de alto riesgo permitieron que el resultado final, en la mayor parte de los problemas neonatales complejos, hoy sea más favorable ${ }^{1}$ : aun cuando han mejorado las tasas de supervivencia, incluso en los niños más inmaduros ${ }^{2}$, tal población sigue siendo vulnerable en cuanto a morbilidad grave y desventajas en el neurodesarrollo a largo plazo. Como consecuencia de ello, las estrategias de estimulación temprana aplicadas por el personal de enfermería se han expandido notablemente y cada vez existe más evidencia sobre la necesidad de su aplicación ${ }^{3}$.

En torno a la estimulación temprana, Rosa Richter de Ayarza la define como "el conjunto de acciones y motivaciones ambientales que se ofrecen al niño desde antes de su nacimiento para ayudarlo a crecer y desarrollarse saludablemente" ${ }^{\text {. }}$.

Vinculado al concepto de estimulación temprana, es necesario referirse a la etapa embrionaria y fetal durante las que se establece las bases del desarrollo del sistema nervioso ${ }^{4}$ : al respecto, Cediel y Blas (2014) (España), en su artículo Utilización del sulfato de magnesio como neuroprotector ante la amenaza de parto prematuro, tuvieron como objetivo identificar la mejor evidencia disponible sobre el tema, cuyo principal resultado fue que en las mujeres con riesgo de parto prematuro inminente las pruebas disponibles indican que administrar sulfato de magnesio intravenoso reduce el riesgo de que el recién nacido sufra parálisis cerebral. Como conclusión queda por determinar el esquema de tratamiento idóneo para lograr la máxima efectividad, con el mínimo riesgo para la salud de la mujer, lo cual se refuerza con otros trabajos publicados que apoyan el uso de sulfato de magnesio como neuroprotector antenatal ${ }^{5}$.

Luego, en enero de 2016, la Editorial Médica Panamericana publicó el libro Neurodesarrollo en Neonatología: Intervención ultratemprana en la Unidad de Cuidados intensivos Neonatales en Buenos Aires, Argentina, por Basso ${ }^{6}$, con el aval de la Federación Internacional NIDCAP: del escrito, se destaca el capítulo III, "Protección del desarrollo en la UCIN", según el cual si bien proteger completamente al recién nacido de todos los factores que pueden interferir con su neurodesarrollo es un ideal, muchas veces inalcanzable, es una responsabilidad ineludible en la actualidad evitar situaciones o tratamientos que afectan de manera adversa al cerebro en desarrollo. El capítulo desarrolla algunas estrategias aplicadas por el personal de enfermería como reducción del estrés, cuidados posturales o manipulaciones gentiles, que promueven la estabilidad fisiológica y estimulan el desarrollo sensoriomotor óptimo, lo cual tiene como resultado una implicancia positiva sobre el neurodesarrollo.

Por otro lado, Capó $^{7}$ en el año 2016 publicó Intervenciones enfermeras sobre el ambiente físico de las unidades de cuidados intensivos en la revista Enfermería Intensiva, en España, cuyo objetivo principal fue analizar las intervenciones enfermeras sobre el ruido y la iluminación que influyen en el neurodesarrollo del neonato prematuro en una unidad de cuidados intensivos neonatal. El trabajo consistió en una revisión bibliográfica que tuvo como resultado principal algunas recomendaciones prácticas como prohibir el uso de teléfonos móviles en la unidad, retirar la televisión y radios, cerrar con suavidad puertas de incubadoras, así como cubrirlas con mantas y otras recomendaciones más, aplicadas al personal de enfermería como hablar con voz suave y baja al neonato y la manipulación mínima. Se concluyó que los enfermeros son los profesionales que más tiempo pasan en el interior de las unidades neonatales y que las intervenciones de concienciación del personal y 


\section{Revista Electrónica Enfermeria Actual en costa Rica}

familia ganan un peso muy importante en la protección de los neonatos, además de que las intervenciones enfermeras deberían potenciarse mucho más en las unidades para así poder influir positivamente en el neurodesarrollo del neonato prematuro.

En Brasil, en el año 2012, Gomes, Mota, Panúncio-Pinto y Lara ${ }^{3}$ llevaron a cabo la investigación titulada Estimulación temprana en enfermería pediátrica: El papel del terapeuta ocupacional, cuyo objetivo fue caracterizar la intervención de terapia ocupacional para la estimulación temprana del desarrollo de niños entre 0 y 2 años de edad, teniendo en cuenta el contexto de la hospitalización. Como resultados se destaca que la enfermera pediátrica realiza las actividades cuando está presente la madre o cuidador e inicia el contacto con el bebé, a través de la conversación, antes de tocarlo, para establecer o retomar el vínculo terapéutico o tranquilizarlo, mientras observa sus respuestas fisiológicas y de comportamiento. La enfermera pediátrica realiza ejercicios de estiramiento muscular, musicoterapia, uso de juguetes musicales y técnicas como balanceo y movimiento en el brazo del adulto. Se favorece además estimulación vestibular y toque terapéutico. Como conclusiones se determina que la estimulación temprana puede ser considerada un método ideal de atención para los bebés hospitalizados con riesgo de atraso en el desarrollo (como lo son claramente los prematuros), el cual no solo depende del terapeuta ocupacional sino también de otros profesionales del equipo multidisciplinario que también acompaña al niño. Además, menciona que el papel del terapeuta ocupacional en enfermería pediátrica es identificar las condiciones, el estado emocional y el comportamiento de los niños, sin olvidar sus limitaciones físicas, así como proporcionar estímulos adecuados para su desarrollo durante y después de su hospitalización.

En 1998, en Brasil, Monte y Freitag ${ }^{8}$ publican Estimulación visual para recién nacidos prematuros, intervención de enfermería, en el que mencionan cómo, desde la concepción hasta el nacimiento, el recién nacido pasa por varios estados del desarrollo hasta alcanzar su complejidad, proceso que es continuo y sufre modificaciones. Por otra parte, agregan que la estimulación sensorial se utilizaba en aquel momento en casos confirmados de trastornos oculares, con el fin de auxiliar en el desarrollo e interacción del individuo al medio en que vive, además de que, aunque los bebés prematuros extremos necesitan protección excesiva, tan pronto como pase ese periodo y presenten mejoría del cuadro clínico deben ser estimulados sensorialmente para que desarrollen su percepción cognitiva y motora. Dicho estudio tuvo como objetivo estudiar la respuesta visual de los neonatos prematuros y, como resultado, se obtuvo que los recién nacidos sometidos a la estimulación visual se mostraron alerta, activos y atentos, mientras que los del grupo control se encontraron desinteresados y poco activos frente al estímulo. Se concluyó que el programa de estimulación permitió ablandar los disturbios visuales por la evaluación constante de las respuestas.

Por otra parte, en julio 2008, Domínguez et $\mathrm{al}^{9}$ publicaron Efectividad de la aplicación de toques terapéuticos en el peso, las complicaciones y los días de estancia, en recién nacidos pretérmino ingresados en una unidad de neonatología, con el objetivo de conocer la efectividad de los toques terapéuticos en el peso, la presentación de complicaciones posnatales y los días de estancia en recién nacidos pretérminos, así como la satisfacción de los padres. Los investigadores utilizaron un estudio experimental (se incluyó $78 \mathrm{RNP}$ ) con grupo control mediante asignación aleatoria y tuvieron como resultados en relación con el peso; la media fue de $1.867,80 \mathrm{~g}$ (desviación estándar [DE] 1/4 149,72) en el grupo experimental y de1.860g (DE 1/4 181,92) en el control $\left(\mathrm{t} 1 / 40,148 ; \mathrm{p}^{1 / 4}\right.$ 0,883); el tiempo de estancia en la unidad fue de 16,82 (DE 1/4 6,47) en el grupo experimental y de 20,30 (DE 1/4 8,04) en el de control ( $1 / 1 / 42,100$, p $\left.1 / 4_{4} 0,039\right)$, y de presentación de complicaciones posnatales, mientras que en el grupo experimental aparecen en el 5,3\% de los RNP,en el grupo control lo hacen en el $20 \%$ 
(w2 1/4 3,78; p 1/4 0,049). La Odds ratio para presentación de complicaciones fue de 1,673 (intervalo de confianza del 95\%, 1,089-2,571). Se concluye que la aplicación de los toques terapéuticos ha mostrado su efectividad en el tiempo de estancia y en la presentación de complicaciones ${ }^{7}$, aunque en futuras investigaciones podrí ampliarse el tamaño de la muestra.

Finalmente, en diciembre 2014, Nair et al ${ }^{1}$ publican el artículo CDC Kerala 2: Developmental Intervention Package for Babies <1,800 g-Outcome at 6 mo Using DASII: su objetivo fue describir la experiencia de usar un paquete de intervención de neurodesarrollo entre bebés con bajo peso al nacer, menor que 1800 gramos y resultado de desarrollo al final de 6 meses de intervención. En cuanto a los resultados, de un total de 821 bebés matriculados por anticipado en el programa de estimulación, 740 completaron con éxito el estudio, 6 meses de seguimiento y programa de estimulación. Al comparar el resultado a los 4 y 6 meses, ambos clasificados por cabeza sostenida y la parte del motor grueso de DDST, se mostró una reducción significativa estadística en hallazgos anormales. Los resultados de este programa de estimulación temprana intensiva para bebés por debajo de $1.800 \mathrm{~g}$ han demostrado la importancia de la intervención temprana mensual usando un paquete de estimulación del desarrollo sistemático orientado.

De acuerdo con los estudios mencionados, se observa la diversidad de estrategias de estimulación que se han establecido para solventar las necesidades de los bebés, no obstante, es importante evidenciar específicamente cuáles estrategias presentan un mejor y más seguro resultado para estos bebes, las cuales puedan ponerse en práctica a nivel hospitalario.

Un nacimiento prematuro es aquel que se produce antes de las 37 semanas completas o 259 días de gestación desde el primer día del último periodo menstrual de la mujer. Así pues, el recién nacido prematuro es el neonato resultante de este nacimiento precoz ${ }^{10}$. Este tipo de nacimientos constituyen un problema de salud importante, ya que, pese a que la esperanza de vida de estos neonatos ha mejorado considerablemente, la morbilidad constituye una preocupación importante para el equipo de salud. Los neonatos prematuros presentan una inmadurez anatómica y funcional importante, sobre todo en el sistema nervioso central, hecho que limita su capacidad para procesar y registrar las informaciones sensoriales y, por tanto, la capacidad de adaptación al ambiente extrauterino. Estas características los hacen especialmente sensibles y vulnerables a estímulos externos ${ }^{7}$.

En cuanto a los recién nacidos pretérminos, una gran cantidad sobrevive cada vez más a la experiencia de la unidad de cuidados intensivos neonatales (UCIN), por lo que es enviada a sus casas con sus padres. El resultado de estos infantes en el neurodesarrollo ha sido ampliamente estudiado ${ }^{11}$, ya que las consecuencias emocionales, afectivas y cognitivas de los niños que nacen con muy bajo peso presentan un problema de salud pública importante.

Debido a que el desarrollo del cerebro se rige en gran medida por estímulos sensoriales, resulta trascendente determinar los cuidados o intervenciones que no solo favorecen el desarrollo del neonato pretérmino, sino que además son útiles para reducir alteraciones en el neurodesarrollo ${ }^{12}$.

En torno a la estimulación temprana, Figueroa mencionado en la página web de Vanesagl ${ }^{4}$ la define como "una serie de actividades efectuadas directamente o indirectamente desde la más temprana edad, dirigidas a proveerle la mayor cantidad posible de oportunidades de interacción efectiva y adecuada con el medio ambiente 
humano y físico, con el fin de estimular su desarrollo general o en áreas específicas". Los recién nacidos pretérmino sufren una interrupción en su neurodesarrollo debido a su nacimiento prematuro, por lo que requieren de una mayor estimulación temprana.

Convenientemente manejada, la estimulación temprana puede producir grandes cambios en el funcionamiento del cerebro y el desarrollo mental, los cuales son mayores en el periodo en que el cerebro crece con más rapidez ${ }^{13}$. A partir del nacimiento el crecimiento es decreciente, en consecuencia, conviene estimular aquella capacidad en los niños nacidos prematuramente, máxime que en ningún período de su vida, el infante desarrolla con mayor rapidez que durante los primeros años de vida: al respecto, cabe mencionar que existe un límite de tiempo para la activación fácil de las células cerebrales, pasado el cual se producirán situaciones tal vez irreversibles, aspecto que refuerza por qué deben ser las enfermeras quienes ofrezcan esta atención temprana a los recién nacidos hospitalizados, no solo por la larga estancia a los que se ven sometidos los neonatos prematuros, sino por pasar la mayor parte del tiempo junto a los pacientes. Lo anterior involucra siempre a los padres, pues el principio básico del cuidado centrado en el paciente y en la familia es que no debe estar solo enfocado en las soluciones técnicas y farmacéuticas del tratamiento, sino que debe ampliarse para abarcar también las necesidades del paciente, de los padres y hermanos ${ }^{6}$.

Este trabajo de investigación es de gran relevancia ya que identificará las intervenciones / actividades de enfermería que integran el gran concepto de estimulación temprana, al determinar sus repercusiones en el cerebro del RNP, órgano de gran importancia en la funcionalidad integral del individuo, ya que las alteraciones en el neurodesarrollo pueden afectar hasta una tercera parte de los pacientes prematuros ${ }^{14}$.

En las unidades de neonatología, en ocasiones, los cuidados de enfermería se prescriben por protocolo ${ }^{15}$ al tratar de resolver un problema inmediato, sin pensar en las consecuencias de tales acciones en el recién nacido, por eso este trabajo aporta una visión novedosa pues consiste en caracterizar esas intervenciones comunes, con la novedad de identificar si promueven o no el neurodesarrollo. Con esa finalidad, se hará una investigación que busque beneficiar al neonato en su etapa posnatal, la cual está cargada de altos niveles de estrés, puesto que ingresa a una unidad de cuidado intensivo neonatal separado de su madre, a lo que se suma un ambiente altamente estresante (ruidos, luces), por lo que también los investigadores pretenden establecer cuál sería el ambiente ideal para un recién nacido prematuro, desde una una visión más integral de las intervenciones de enfermería, no solo en cuidado directo, sino indirecto.

Las intervenciones centradas en la familia que favorecen el desarrollo reducen el estrés ${ }^{16}$ y proporcionan un tratamiento ante el dolor, además de que favorecen la autorregulación del infante, promueven la presencia, el vínculo y el apego de los padres, lo que disminuirá el estrés parental, que mejore el desarrollo cerebral ${ }^{17} \mathrm{y}$ afecte de manera positiva los desarrollos cognitivo y psicomotor del infante ${ }^{18}$.

En relación con lo expuesto, la práctica basada en evidencia (PBE) es un enfoque de solución de problemas para la práctica ${ }^{19}$ clínica $^{\mathrm{i}}$ que integra la mejor evidencia científica e información del paciente con la experticia clínica, así como las preferencias y valores de los pacientes ${ }^{20}$. 
Como futuros especialistas en enfermería pediátrica, es de gran relevancia clínica determinar qué estrategias de estimulación o atención temprana (como más recientemente se maneja el concepto) tienen un impacto positivo en el neurodesarrollo del recién nacido pretérmino.

El objetivo de este protocolo es analizar la mejor evidencia disponible respecto de las estrategias de estimulación temprana, aplicadas por enfermería a los recién nacidos pretérmino hospitalizados, que han sido útiles para reducir o evitar alteraciones leves o graves en el neurodesarrollo.

\section{MÉTODO}

Se aplica la metodología de práctica clínica basada en la evidencia, compuesta por cinco etapas: 1. Elaboración de la pregunta clínica 2. Búsqueda información científica 3. Análisis crítico de la mejor evidencia encontrada 4. Transferencia de la evidencia científica y 5. Evaluación de la transferencia de la evidencia científica.

\section{Pregunta clínica}

La pregunta clínica elaborada es ¿qué tipo de estrategias de estimulación temprana aplicadas por enfermería han sido útiles para reducir o evitar alteraciones leves o graves en el neurodesarrollo de los recién nacidos de pretérmino hospitalizados? Para el planteamiento de la pregunta se utilizó el acrónimo PCC (población, concepto y contexto)

Población: Recién nacidos pretérmino

Concepto: Estrategias de estimulación temprana

Contexto: Unidad de cuidados intensivos neonatales

El tipo de pregunta se clasifica como una pregunta de "alcance", la cual se elabora cuando se quiere determinar el alcance de un cuerpo de literatura sobre un tema. Se utiliza para mapear los conceptos clave que sustentan un área de investigación, aclarar las definiciones de trabajo y /o los límites conceptuales de un tema e identificar brechas en la base de conocimientos ${ }^{21}$.

\section{Criterios de inclusión}

Los criterios de inclusión para recuperar la evidencia son los siguientes:

Población: se considerará estudios que incluyan recién nacidos pretérmino hospitalizados independientemente de los días de nacido, sexo o edad gestacional.

Concepto: se incluirá estudios que hagan referencia a los recién nacidos pretérmino que hayan recibido estimulación temprana por parte de enfermería y que no presenten alteraciones en la salud evidentes que comprometan su neurodesarrollo.

Contexto: se tomará en cuenta estudios vinculados a los recién nacidos pretérmino que estén hospitalizados. 


\section{Tipos de estudios}

Se considerará los estudios publicados tanto experimentales como no experimentales; los diseños incluyen ensayos clínicos, ensayos clínicos aleatorizados, series de tiempo; además, estudios observacionales que incluyen cohortes prospectivos y retrospectivos, casos y controles y estudios descriptivos que se complementa con reportes de casos individuales, estudios con diseño cualitativo, cuya publicación esté en idioma español, inglés y portugués.

\section{Estrategia de búsqueda}

La estrategia de búsqueda tendrá como objetivo encontrar estudios publicados, siguiendo tres pasos: inicialmente se llevará a cabo una búsqueda limitada en MedLine, Google Scholar, Science direct, EBSCO seguida de un análisis de las palabras textuales contenidas en el título y el resumen, y de los términos de índice utilizados para describir cada artículo. Luego se realizará una segunda búsqueda utilizando para ello los descriptores planteados en esta revisión en las bases de datos PubMed, Cochrane Plus, Medline, Science Direct, Scielo, Clinical Key, Ovid y otros recursos para búsqueda de literatura como SIBDI (UCR) y BINASS, para lo que se solicitó ayuda para la recuperación de artículos científicos relacionados al tema. En tercer lugar, se buscará estudios adicionales en la lista de referencias de todos los informes y artículos identificados. Se incluirá toda la información disponible entre los años 2012 y 2017.

\section{Descriptores de búsqueda}

Para la búsqueda de información se utilizará los descriptores, en idioma español, inglés y portugués expuestos en la tabla1, utilizando para ello los boléanos AND, OR, NOT.

Tabla 1. Descriptores para la búsqueda de información

\begin{tabular}{l|l|l}
\hline \multicolumn{1}{c}{ Español } & \multicolumn{1}{c}{ Inglés } & \multicolumn{1}{c}{ Portugués } \\
\hline Recién nacido & Newborn & Recém nascido \\
\hline Pretérmino & Preterm & Pré-termo \\
\hline Enfermería & Nursing & Enfermagem \\
\hline Neurodesarrollo & Neurodevelopmental & Desenvolvimento neurológico \\
\hline Estimulación temprana & Earlier stimulation & Estimulação precoce \\
\hline Atención temprana & Earlier attention & Cuidado precoce \\
\hline
\end{tabular}

Fuente: elaboración propia 


\section{Revista Electrónica Enfermeria Actual en costa Rica}

\section{Selección de los estudios}

Luego de la búsqueda de información, todas las citas identificadas se recopilará y se cargará en el software bibliográfico Mendeley y se eliminará los duplicados. Se examinará los títulos y los resúmenes para evaluarlos según los criterios de inclusión para la revisión. Los estudios que cumplan con los criterios de inclusión se recuperará en su totalidad y se importará sus detalles en una matriz de datos de Excel. El texto completo de los estudios seleccionados se recuperará y evaluará en detalle en relación con los criterios de inclusión. Se excluirá los estudios de texto completo que no cumplan con los criterios de inclusión y se proporcionará los motivos de la exclusión en un apéndice del informe de la revisión final.

Los resultados de la búsqueda se anotará en su totalidad en el informe final y se presentará en un diagrama de flujo PRISMA 2009 en su versión en español.

Cualquier desacuerdo que surja entre los investigadores se resolverá mediante una discusión conjunta.

\section{Extracción de los datos}

Se extraerá los datos de los artículos incluidos en la revisión de forma manual, los cuales incluirán detalles específicos, como año, autor, título, tipo y métodos del estudio, participantes, tipo de intervención, resultado principal y secundario de importancia para la pregunta clínica planteada y los objetivos específicos.

Cualquier desacuerdo entre los investigadores se resolverá mediante una discusión conjunta. Se contactará a los autores de los documentos para solicitar datos faltantes o adicionales cuando sea necesario.

\section{Evaluación de la calidad metodológica}

Para la evaluación de la calidad metodológica se hará análisis crítico de la evidencia ${ }^{21}$, el cual consiste en seleccionar información de calidad y utilizable en la investigación, mediante herramientas que ayudan y permiten determinar si un estudio es válido o no para ser usado. Al investigar, es sumamente importante realizar este paso ya que los resultados de las investigaciones y todo el trabajo en sí y su calidad, dependerá de la información que se reúna. Hay investigaciones que contienen datos no confiables, y si no se evalúan para decidir si se seleccionan, podrían acarrear errores durante y luego del proceso investigativo, ya que muchos de los estudios son usados como referencias para elaborar guías o protocolos de acción y, como mencionan Rojas, Cubero y Leiva ${ }^{21}$ “el actuar con base en las pruebas científicas inválidas o incorrectas es probablemente peor que actuar sin pruebas. Además, en el mejor de los casos la práctica se distorsiona de manera inconsciente y en el peor de los casos puede causar daño a las y los pacientes".

Respecto de la lectura crítica Rojas, Cubero y Leiva ${ }^{21}$ exponen que es

"el proceso de evaluar e interpretar la evidencia aportada por la bibliografía científica, considerando sistemáticamente los resultados que presentan, su validez y su relevancia para el propio trabajo..., a través de la lectura crítica se verifican la validez y la aplicabilidad de los resultados publicados con el fin de poder incorporarlos al cuidado de nuestros pacientes". 


\section{Revista Electrónica Enfermeria Actual en costa Rica}

Existen otras herramientas que ayudan a seleccionar cuáles documentos son válidos y confiables, como por ejemplo la plataforma de fichas de lectura crítica 2.0 (FLC 2.0), la cual se encuentra en internet, es libre y gratuita y permite ir reuniendo o almacenando los artículos que se van revisando. La plataforma va dirigiendo la lectura crítica paso por paso, para al final determinar si el artículo tiene alto, moderado, bajo o muy bajo nivel de calidad. Por otro lado, también se puede utilizar el Critical Appraisal Skills Programme Español (CASPe) que proporciona una serie de plantillas según el tipo de estudio que se evalúe para ayudar al investigador a emitir un criterio respecto del documento evaluado en términos de calidad y validez o el AGREE II que se utiliza para evaluar guías de práctica clínica.

En el presente trabajo, se utilizará la plataforma FLC 2.0 y las plantillas CASPe para el análisis crítico de la evidencia, y así determinar la calidad, validez y confiablidad o si hay sesgos en el documento seleccionado para análisis crítico.

\section{Síntesis de datos}

Los resultados de esta revisión breve serán sintetizados y presentados en forma de tabla de evidencia y resumen narrativo de acuerdo con los objetivos específicos para su análisis posterior.

\section{Conflicto de intereses}

Los autores declaran que no tienen conflictos de tipo personal, comercial, académico, político o financiero.

\section{Agradecimientos}

Los autores del presente artículo agradecen a las profesoras del curso de investigación de Enfermería Basada en la Evidencia Ligia Patricia Rojas Valenciano y Consuelo Cubero Alpízar, por su guía durante la elaboración. De la misma manera, se extiende un agradecimiento al comité de investigación de la Escuela de Enfermería, por sus aportes y sugerencias durante el camino.

\section{REFERENCIAS BIBLIOGRÁFICAS}

1. Nair et al. CDC Kerala 2: Development Intervention package for babies $<1.800 \mathrm{~g}$ - Outcome at 6 mo Using ASIII. Indian J Pediatr (India). 2014; 81 : S73-S79

2. Ho YB, Lee RS, Chow CB, Pang MY. Impact of massage therapy on motor outcomes in very low-birthweight infants: Randomized controlled pilot study. Pediatr Int. 2010;52(3):378-85. doi: 10.1111/j.1442200X.2009.02964.X

3. Gomes da Cunha P, Mota A, Panúncio MP, Lara L. Estimulación temprana en enfermería pediátrica: El papel del terapeuta ocupacional. TOG. 2012;16. 
4. Vanesagl. Modelos y Prácticas educativas. Grado Educación Infantil. Disponible en https://vanesagl.wordpress.com/2014/05/15/estimulacion-temprana/

5. Rohlfs P. Desarrollo del sistema nervioso humano. Perspectiva general del estadio prenatal hasta 2013. Revista Internacional de Psicología. 2016; 15(1).

6. Basso G. Neurodesarrollo en Neonatología. Intervención ultratemprana en la unidad de cuidados intensivos neonatales. Editorial panamericana. 2016.

7. Capó M. Intervenciones enfermeras sobre el ambiente físico de las Unidades de Cuidados Intensivos Neonatales. Enfermería Intensiva. 2016; 27( 3):96-111.

8. Sousa KM, Pagliuca, LMF. Estimulación visual para recién nacidos prematuros, Intervención de Enfermería. $R$. Bras. Enferm. 1998;51 (2):, p. 189-206. http://dx.doi.org/10.1590/S0034-71671998000200002.

9. Domínguez Rosales et al. Efectividad de la aplicación de toques terapéuticos en el peso, las complicaciones y los días de estancia, en recién nacidos pretérmino ingresados en una unidad de neonatología. Enfermería Clínica. 2009;19( 1): 11-15. https://doi.org/10.1016/j.enfcli.2008.07.001

10. Beck S, Wojdyla D, Say L, Betran AP, Merialdi M, Requejo JH, et al. The worldwide incidence of preterm birth: a systematic review of maternal mortality and morbidity. Bull World Health Organ. 2010;88 (1:S2).

DOI:10.2471/BLT.08.062554

11. Vizcaíno, MCR. Atención temprana: recursos y pautas de intervención en niños de riesgo o con retrasos del desarrollo. Asociación Española de pediatría. España: 278-285.

12. Valle M, Sierra P, Palacios I, García R, Moro M. Atención temprana al recién nacido de riesgo. An Pediatr Contin. 2014;12(3):119-23.

13. Orós D. Perinatal and neurodevelopmental outcome of late-onset growth restricted fetuses. [Tesis Doctoral]. Universitat De Barcelona. Departament D'Obstetrícia I Ginecologia, Pediatria I Radiologia I Medicina Física 2010

14. Cordero G. Santillan V, Carrera S, Corral E, Fernández LA. Estrategias de ventilación a favor de la neuroprotección: ¿qué podemos hacer? Perinatología y Reproducción humana. 2016; 30(3): 130-137. https://doi.org/10.1016/j.rprh.2016.10.006

15. Sevilla ER, Rizatto DM. Evaluación del protocolo de identificación de los recién nacidos en un hospital privado. Revista Latino-Americana de Enfermagem. 2012; 20(4): 659-667. 
16. Villamizar, B. Vargas, C. Gómez-Ortega Olga. Metaanálisis: efecto de las intervenciones para disminuir el nivel de estrés en padres de prematuros. Aquichan. 2016; 16(3):276-295

17. Als H, Duffy FH, McAnulty G, Butler SC, Lightbody L, Kosta S, et al. NIDCAP improves brain function and structure in preterm infants with severe intrauterine growth restriction. Journal of perinatology: official journal of the California Perinatal Association. 2012 Oct; 32(10):797-803.

18. Montirosso R, Del Prete A, Bellu R, Tronick E, Borgatti R. Level of NICU quality of devepmental care and neurobehavioral performance in very preterm infants. Pediatrics. 2012 may; 129 (5): e1129-37.

19. Ramos De La Cruz, Ediltrudis. Enfermería basada en la evidencia. Duazary, 01 December 2013, Vol.1(2)

20. Melnyk BM, Fineout-Overholt E, Stillwell SB, Williamson KM. Evidence-based practice: step by step: Igniting a spirit of inquiry: an essential foundation for evidence-based practice. Am J Nurs. 2009 Nov; 109 (11): 49-52. Doi: 10.1097/01.NAJ.0000363354.53883.58 Disponible en https://www.ncbi.nlm.nih.gov/pubmed/19858857

21. Rojas, P., Cubero, C., Leiva, V. Investigación secundaria: utilización de la mejor evidencia científica en la práctica clínica. 3era Edición. San José, Costa Rica. (2017).

22. Ramos De La Cruz, Ediltrudis. Enfermería basada en la evidencia. Duazary, 01 December 2013, Vol.1(2) 